\title{
Appendicitis presenting with left flank pain
}

\begin{abstract}
Appendicitis is a common surgical condition, whereby diagnosis is usually based on thorough history and examination, alongside routine blood tests. This is a report of acute appendicitis presenting with left flank pain. A 26year-old overweight female presented with one day of stabbing left loin to groin pain of increasing in severity. The initial diagnosis of renal colic was ruled out with ultrasound of the kidneys, ureters and bladder. She then developed severe sepsis, with raised inflammatory markers. An urgent $\mathrm{CT}$ abdomen/pelvis was suggestive of appendicitis.
\end{abstract}

She underwent an emergency laparoscopy, washout and appendicectomy, with histopathology in keeping with acute suppurative appendicitis with necrosis and peritonitis. Appendicitis presenting with left flank pain has only been reported once in the literature. The varying presenting symptoms are thought to be due to the position of the appendix- in this case, a pelvic appendix also involving the small bowel with reported perforation risk reported to rise with increasing diagnostic delay, prompt diagnosis and treatment is necessary to prevent any unnecessary morbidity or mortality.
Volume 4 Issue 3 - 2016

\author{
Lolade Giwa,' Adenike Odeleye, ${ }^{2}$ Abraham \\ Ayantunde 3 \\ 'Department of Surgery, Southend University Hospital, UK \\ ${ }^{2}$ Accident and Emergency, Newham Hospital, UK \\ ${ }^{3}$ Department of Surgery, Southend University Hospital, UK
}

\section{Correspondence: Lolade Giwa, Department of Surgery, Southend University Hospital, Prittlewell Chase, Westcliff-on- Sea, SSO ORY,Tel 01702435555, Fax 0170252 I32I,}

Email lolagiwa@doctors.org.uk

Received: October 26, 2015 | Published: April 12, 2016

Keywords: acute appendicitis, left flank pain, abdominal pain

Abbreviations: CT, computed tomography scan; WBC, white blood cells; BP, blood pressure; CRP, C-reactive protein; RIF, right iliac fossa: RUQ, right upper quadrant

\section{Introduction}

Appendicitis is a common surgical condition, accounting for 40000 hospital admissions per year. ${ }^{1}$ Typical presenting symptoms of appendicitis, originally described by Murphy, of central abdominal pain due to mid gut visceral irritation migrating to the right iliac fossa as the inflammation progresses alongside vomiting may not be as common as teaching may indicate. Murphy et al., ${ }^{2}$ found these symptoms only to be present in $50 \%$ people diagnosed with acute appendicitis.

Despite this, appendicitis does generally present with right sided pain being one of the key features and so a patient presenting with left sided flank pain alone- a key feature of renal colic is incredibly unusual. The fact that the patient became rather sick rather quickly shows that right sided pain is not one of the only things one should be looking for when diagnosing appendicitis.

\section{Case presentation}

A 26year old woman presented with severe left flank pain. She described a one-day history of stabbing left loin-to-groin pain waking her from sleep, which was constant in nature and of increasing severity. The pain was worse on movement. She reported fever and having vomited about 6 times prior to presentation. The patient had experienced a self-limiting episode of bloody diarrhea and abdominal pain 4days prior to presentation. The patient reported a 'few days' of urinary frequency, and one day history of low volume, dysuria and very foul smelling urine. Relevant past medical history revealed polycystic ovarian syndrome.

Clinical examination showed an acutely unwell patient with lowgrade pyrexia, dehydration, tachycardia with significant abdominal tenderness worst in the left flank. There was not undue tenderness in the right iliac fossa. Urinalysis revealed cloudy, concentrated urine with only a trace of protein and $\beta-\mathrm{HCG}$ was negative.
Routine blood tests showed raised leucocytes of $15.4 \times 104$ with a neutrophilia $(12 \times 104)$. The rest of the blood test results were unremarkable, with a C-reactive protein of 2. Differential diagnoses of sterile pyelonephritis/renal colic were made. An ultrasound of the urinary tract was normal.

Overnight, the patient became septic with WBC 17.5(15), SBP 90 109 systolic, heart rate 130, rigors, Temperature 39.3, CRP 202. By this time, the abdomen was diffusely tender with severe tenderness at both flanks. An urgent CT abdomen/pelvis showed some inflammatory looking changes in the right iliac fossa with a distended appendix and an appendicolith; in keeping with appendicitis.

She underwent an emergency laparoscopy, washout and appendicectomy. Operative findings were of a necrotic appendix with an inflammatory mass including the omentum and small bowel. There was a normal terminal ileum, ovaries and uterus. There a 21 wash to the RIF, pelvis and RUQ and a drain was left in situ.

The patient spent 4days in hospital on intravenous antibiotics. The histology of the appendix was in keeping with acute suppurative appendicitis with necrosis and peritonitis. The patient was discharged 4days post-operatively and is well, with no further attendances to the hospital.

\section{Discussion}

Typical presenting symptoms of appendicitis, originally described by Murphy, of central abdominal pain due to mid gut visceral irritation migrating to the right iliac fossa as the inflammation progresses alongside vomiting may not be as common as teaching may indicate. Murphy et al. found these symptoms only to be present in $50 \%$ people diagnosed with acute appendicitis. ${ }^{2}$

Presenting symptoms of appendicitis can depend on the lie of the appendix. A retrocaecal appendix (75\%) may present with signs of psoas irritation alongside the RIF pain, pelvic appendixes (20\%) can present with urinary tract symptoms or diarrhea due to irritation of the ureters/ bladder and rectum respectively. An appendix closely related to the ileum (5\%) may present with symptoms of ilieal irritation, such 
as diarrhea and vomiting. ${ }^{3}$ It is possible that in this patient, a pelvic appendix may have caused bladder irritation, irritating the ureters, leading to the diffuse abdominal and loin pain. The earlier bout of bloody diarrhea may have been early signs of an inflamed appendix making contact with the ileum/rectum.

The varied reliability of imaging modalities is further highlighted in our case, as our patient did actually undergo an ultrasound of the abdomen, which did not pick up any signs of acute appendicitis. The role CT played in this picture was not in the normal setting of diagnosing appendicitis, but in diagnosing an unknown intraabdominal inflammatory pathology.

The Alvarado Score was constructed to predict likelihood of a diagnosis of appendicitis based on a set of diagnostic markersbiochemical and physical. The use of the Alvarado score has fallen from popularity and a pilot study in Sheffield has shown poor correlation of Alvarado score with positive appendicitis diagnosis, ${ }^{4}$ alongside other studies.

The patient in question had a score of $7 / 10$, which would indicate probable appendicitis, however the benefit of hindsight may play a role in achieving this score, as although RIF pain was present, this was not the point of maximal pain.

In a meta-analysis of the signs and symptoms of appendicitis, central abdominal pain migrating to the RIF was the only symptom found to be reliably associated. ${ }^{5}$ This symptom was not present in our patient, demonstrating the potential diagnostic difficulties in appendicitis.

Appendicitis with left flank pain has been reported once in the literature in a 42-year-old woman with urinary tract symptoms and positive urinalysis. ${ }^{6}$ An incidental appendicolith was seen on CTsimilarly to this reported case, but no other signs of RIF inflammation were seen. This patient, however, was treated successfully with antibiotics and represented one week later with typical appendicitis- like pain. The authors discussed whether the initial presentation was simply a red herring and unrelated to the appendicitis.

With reported perforation risk reported to rise by $5 \%$ for every 12 hours after the first 36 hour period after onset of symptoms, ${ }^{7}$ prompt diagnosis and treatment is necessary to prevent any unnecessary morbidity or mortality. Other studies have also noted that a delay to diagnosis past the initial 24/36hours resulted in poorer outcomes. ${ }^{8}$

\section{Acknowledgements}

None.

\section{Conflict of interest}

The author declares no conflict of interest.

\section{References}

1. Hospital episode statistics. Primary diagnosis: summary.

2. Murphy J. Two thousand operations for appendicitis, with deductions from his personal experience. Am J Med Sci. 1904;128:187-211.

3. Humes DJ, Simpson J. Acute appendicitis. BMJ. 2006;333(7567):530534.

4. Lee J, Rudge E, Boyes S. Pilot study: evaluation of alvarado score in the diagnosis of acute appendicitis - a retrospective data analysis. Int J Surg. 2013;11(8):709-710

5. Andersson RE. Meta-analysis of the clinical and laboratory diagnosis of appendicitis. Br J Surg. 2004;91(1):28-37.

6. Talanow R. An unusual manifestation of acute appendicitis with left flank pain. J Radiol Case Rep. 2008;2(1):8-11.

7. Bickell NA, Aufses AH, Rojas M, et al. How time affects the risk of rupture in appendicitis. J Am Coll Surg. 2006;202(3):401-406.

8. Papaziogas B, Tsiaousis P, Koutelidakis I, et al. Effect of time on risk of perforation in acute appendicitis. Acta Chir Belg. 2009;109(1):75-80. 